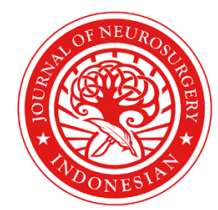

\title{
Analysis of Intracranial Aneurysm Detection using Time-based Parametric Color-Coded Cerebral Angiogram Vs. Conventional Digital Subtraction Angiography
}

\author{
Achmad Adam, ${ }^{1}$ Ahmad Faried, ${ }^{1 *}$ Wargian Hadisaputra, ${ }^{1}$ Beni Rio Hermanto, ${ }^{2}$ \\ Muhammad Zafrullah Arifin ${ }^{1}$
}

\section{ABSTRACT}

Introduction: The worldwide prevalence of intracranial aneurysms is estimated to be 5-10\%; approximately 10-12 million people are affected by intracranial aneurysms in the United States (US) and they are considered a major problem in public health. The manual interpretation of intracranial aneurysms based on visual examinations and direct observation of medical imaging techniques such as computed tomography (CT) scans, magnetic resonance imaging (MRI), or any of the digital subtraction angiography (DSA), are still presented in the monochrome form. Thus, it takes time to interpret the medical images.

Methods: Alternative method is still being developed, allowing detection of intracranial aneurysms using open source MATLAB software. This method is a way to observe the aneurysm using the 2 dimensional (2D) DSA data based on the calculations of time to peak (TTP) and time duration (TD) of the contrast agent flow in the blood vessel in order to capture the aneurysm and visualize it using a color system based on a specific index.

Results: In this study, the color changes of the DSA data that were processed using open source MATLAB software were found in 24 cases $(88.9 \%)$ and only 3 cases $(11.1 \%)$ did not show any color changes. Based on Spearman's correlation test, there was a significant correlation between the size of the high dome, dome-neck ratio, and the aspect ratio $(p<0.01)$ on the color changes of data that were processed using DSA MATLAB software.

Conclusion: The modification methods capturing color changes in different blood vessels are expected to be able to assist clinicians in the detection of intracranial aneurysms quicker and more precisely and are also highly dependent on the subjectivity of the observer; this method is a way to help neurosurgeons come up with new approaches for the management of aneurysms.

Keywords: Intracranial aneurysm, DSA, MATLAB software, color changes

Cite This Article: Adam, A., Faried, A., Hadisaputra, W., Hermanto, B.R., Arifin, M.Z. 2018. Analysis of Intracranial Aneurysm Detection using Time-based Parametric Color-Coded Cerebral Angiogram Vs. Conventional Digital Subtraction Angiography. Indonesian Journal of Neurosurgery 1(1): 23-27. D01:10.15562/ijn.v1i1.1

'Department of Neurosurgery, Faculty of Medicine, Universitas Padjadjaran - Dr. Hasan Sadikin Hospital, Bandung, Indonesia; ${ }^{2}$ Department of Biomedical Engineering, Bandung Institute of Technology, Bandung, Indonesia.

${ }^{*}$ Correspondence to: Ahmad Faried, MD., PhD. Department of Neurosurgery, Faculty of Medicine, Universitas Padjadjaran - Dr. Hasan Sadikin Hospital, Jl. Pasteur No. 38, Bandung 40161, West Java, Indonesia. faried.fkup@gmail.com

\section{INTRODUCTION}

The estimated worldwide prevalence of intracranial aneurysms is $5 \%-10 \%$. In the general, approximately around 10-12 million people are affected by intracranial aneurysms in the United States (US), as there group are the majority caused problem in public health point of view. ${ }^{1}$ Patients with no history of trauma, the intracranial aneurysm is a major cause of subarachnoid bleeding (SAB), which accounts for approximately $85 \%$ of cases. ${ }^{2}$ $\mathrm{SAB}$ due to intracranial aneurysms account for approximately $3-11 \%$ of overall stroke incidences and $5 \%$ of stroke deaths. This disease causes the patient to lose more than a quarter of his/her future productive life, as the disease often occurs during the reproductive age. ${ }^{3}$

In Indonesia, there is no national epidemiological data on the incidence of SAB yet. From 20082009 , in Surabaya, SAB was diagnosed in as many as 37 cases, and 17 cases of surgery were performed within them. This number is very small compared to neighboring countries such as Malaysia and Thailand. Based on the global literature, when the number of events is adjusted to Indonesia's population of 250 million, SAB will be diagnosed in more than 20,000 cases per year on a national scale. ${ }^{4}$

The diagnosis of ruptured SAB due to intracranial aneurysms can be made based on clinical suspicion, supported by findings of bleeding along with its location on computed tomography (CT) scans of the head. The manual interpretation of intracranial aneurysms based on visual examinations and direct observation of medical imaging techniques such as computed tomography (CT) scans, magnetic resonance imaging (MRI), or any of the digital subtraction angiography (DSA), are still presented in the monochrome form and take time for interpretation of the medical images (again, this sentence is repeating the abstract, try to 
paraphrase it). More effective methods are needed in order to diagnose intracranial aneurysms that are not relying on the subjectivity of the observer.

This study attempts to develop and analyze an alternative method that could help to detect intracranial aneurysms using an open source MATLAB software by visualizing the aneurysm in the two-dimensional (2D) DSA data and translating it into specific colors. This method is based on the calculation of whether the contrast agent flow in the blood vessels is impaired or whether there is turbulence, based on the time it takes to reach the peak point (Time to Peak - TTP), and the length of time it is in the blood vessels (Time Duration - TD).

\section{METHODS}

The target population consisted of patients with intracranial aneurysms with saccular type whom confirmed by DSA from January 1 to December 31, 2016; 27 patients that were admitted to Dr. Hasan Sadikin Hospital, Bandung, Indonesia and they were recruited from a satellite hospital. The blood velocity in human blood vessels can be calculated using a Poiseuille equation. ${ }^{5}$ Abnormal types of blood vessels, such as atherosclerotic vessels, produced a turbulent flow compared to the normal vessel that produced a streamlined flow; this is known as Reynolds Number (Re), ${ }^{6}$ with the normal human blood flow at 37 it is $\mathrm{Re}=\bar{v} . \mathrm{D} / 0.027 .{ }^{7}$ The calculation of the TTP value was based on the pixel

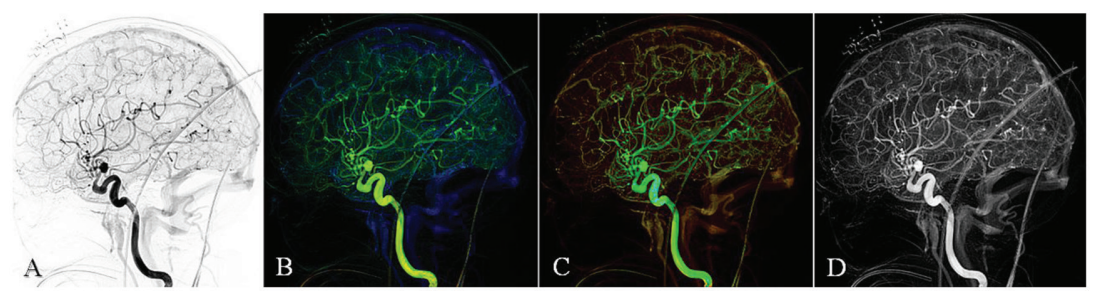

Figure 1 Minimum intensity projection (MIP) for 2D monochrome-image segmentation (A); shown calculation on time to peak (TTP) (B); shown calculation on time duration (TD) (C); maximum differential projection (MDP) in the same case (D)

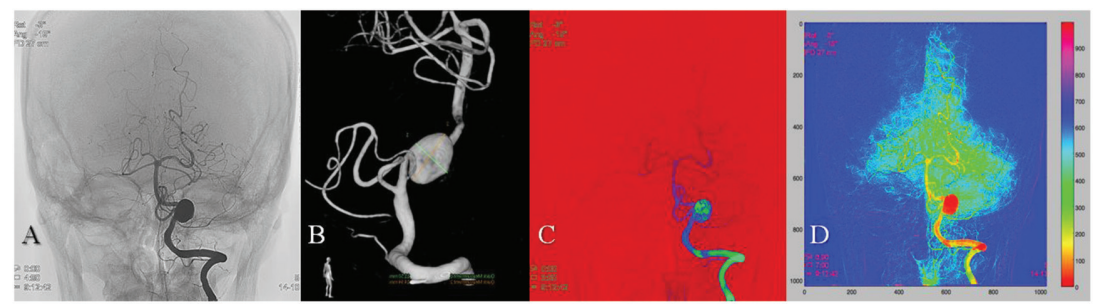

Figure 2 Visualization-based MIP 2D monochrome-image segmentation case no. 11 (A). Shown on the right posterior communicant artery for 3D reconstruction (B). Shows calculation on TTP (C) and combination with time duration using filter multi-scale vessel enhancement filtering to improve the vessel segmentation process (D) intensity of minimum intensity projection (MIP) images (Figure 1A-1C) and for TD, it was based on the number of frames when the pixel intensity falls below the threshold. Image segmentation was used to extract the structure of the blood vessels. There were two steps in this process, which were the calculation of the area of interest and the blood vessel segmentation. The area of interest was selected manually in order to remove the unnecessary area on the image before a multi-scale vessel enhancement filtering (MVEF) method was used to improve the vessel segmentation process (Figure 2A-2C), as described previously. ${ }^{8}$ Data were analyzed using open source MATLAB, using a high-level programming language that is devoted to the needs of technical computing, visualization and programming, such as mathematical computation, data analysis, algorithm development, simulation and modeling, and graphics calculations. MATLAB comes with different staining options since it works best in the functions of mathematics, physics, statistics, and visualization. MATLAB was developed by MathWorks, which was originally launched to provide easier data access on the project matrix LINPACK and EISPACK. Currently, MATLAB has hundreds of functions that can be used for problem-solving, ranging from simple to complex problems and across various disciplines. ${ }^{9,10}$

\section{RESULTS}

This study is a descriptive analysis of a prospective correlation with characteristics of the study subjects consisted of gender, age, location and type of aneurysms. Aneurysm classification was based on the size of the width of the dome, the absence of ruptured aneurysm, the presence or absence of vasospasm, patient complaint upon entry to the hospital, Fisher Grade, and Hunt and Hess score.

The subjects included in this study were almost balanced in terms of gender, with 13 men (48\%) and 14 women (52\%). Thirteen patients with intracranial aneurysms were between the ages of 50-59 years (48\%). In terms of location, 7 cases of aneurysms were found in the area of the internal carotid artery (26\%). As many as 23 cases of aneurysms were small aneurysms (85\%). Of the total patients with intracranial aneurysms, only 4 cases (15\%) were ruptured with only one case experienced vasospasm ( $4 \%$ of the total cases). Eighty-five percent of the study subjects came to the hospital with no symptoms and their aneurysms were found incidentally during a routine check-up. Based on the assessment of blood accumulation in the head CT-scan and the degree of Fisher, majority of the patients $(89 \%)$ had the first degree or no blood found (Table 1). Based on Table 2, the color 
Table 1 Aneurysm patient's characteristics

\begin{tabular}{|c|c|c|}
\hline \multicolumn{2}{|l|}{ Characteristics } & \multirow{2}{*}{$\frac{\mathbf{N}=\mathbf{2 7}(\%)}{13(48)}$} \\
\hline Gender: & Male & \\
\hline & Female & $14(52)$ \\
\hline \multirow[t]{5}{*}{ Age : } & 30-39 years old (YO) & $2(7)$ \\
\hline & $40-49 \mathrm{YO}$ & $4(15)$ \\
\hline & $50-59 \mathrm{YO}$ & $13(48)$ \\
\hline & $60-69 \mathrm{YO}$ & $4(15)$ \\
\hline & $>70 \mathrm{YO}$ & $4(15)$ \\
\hline \multirow[t]{7}{*}{ Aneurysm location : } & $\mathrm{ACA}$ & $4(15)$ \\
\hline & AComA & $4(15)$ \\
\hline & AChoA & $2(7)$ \\
\hline & ICA & $7(26)$ \\
\hline & MCA & $6(23)$ \\
\hline & PComA & $2(7)$ \\
\hline & VA & $2(7)$ \\
\hline \multirow[t]{3}{*}{ Aneurysm size : } & Small & $23(85)$ \\
\hline & Large & $3(11)$ \\
\hline & Giant & $1(4)$ \\
\hline \multirow[t]{2}{*}{ Aneurysm rupture : } & Yes & $4(15)$ \\
\hline & No & $23(85)$ \\
\hline \multirow[t]{2}{*}{ Vasospasm : } & Yes & $1(4)$ \\
\hline & No & $26(96)$ \\
\hline \multirow[t]{3}{*}{ Chief complaint: } & Nothing & $23(85)$ \\
\hline & Headache & $1(4)$ \\
\hline & Decrease of consciousness & $3(11)$ \\
\hline \multirow[t]{4}{*}{ Fisher Grade : } & 1 & $24(89)$ \\
\hline & 2 & $0(0)$ \\
\hline & 3 & $1(4)$ \\
\hline & 4 & $2(7)$ \\
\hline \multirow[t]{6}{*}{ Hunt and Hess Grade : } & 0 & $23(84)$ \\
\hline & 1 & $1(4)$ \\
\hline & 2 & $0(0)$ \\
\hline & 3 & $1(4)$ \\
\hline & 4 & $1(4)$ \\
\hline & 5 & $1(4)$ \\
\hline
\end{tabular}

Note: Anterior Cerebral Artery (ACA), Anterior Communicating Artery (AComA), Anterior Chroidal Artery (AChoA), Internal Carotid Artery (ICA), Medial Cerebral Artery (MCA), Posterior Communicating Artery (PComA), Vertebral Artery

Table 2 Color changing DSA data on software MATLAB

\begin{tabular}{llcc}
\hline & & Frequency & Percentage \\
\hline Color changing DSA & Yes & 3 & 11,1 \\
& No & 24 & 88,9 \\
& Total & 27 & 100,0 \\
\hline
\end{tabular}

Note: Digital Subtraction Angiography

Table 3 Statistic description on aneurysm size's morphology

\begin{tabular}{lccc}
\hline Variables & Mean \pm SD & Minimum Value & Maximum Value \\
\hline Neck Wide & $3,9 \pm 3,0$ & 1,12 & 15,00 \\
Dome Wide & $5,2 \pm 4,1$ & 1,35 & 20,00 \\
Dome Height & $5,3 \pm 5,8$ & 1,48 & 29,00 \\
Dome Neck Ratio & $1,4 \pm 0,53$ & 0,60 & 2,68 \\
Aspect Ratio & $1,3 \pm 0,6$ & 0,43 & 2,63 \\
\hline
\end{tabular}

changed on the DSA data that was processed using MATLAB software in 24 cases (88.9\%), and only 3 cases $(11.1 \%)$ did not show any color changing. Additionally, the authors tried to find a correlation between the variables, but there was no correlation found regarding the color changes.

The variables that were tested for statistical correlation regarding aneurysm morphology included: wide neck, wide dome, high dome, dome-neck ratio, and aspect ratio. In this study, data processing of the aneurysm DSA was already completed via image segmentation. Furthermore, a filtration image was used to improve accuracy and to reduce the factors that may be biased when processing the image data. From the processed picture, the value of both the aneurysm anatomy and morphology can be measured. Regarding the morphological characteristics of the aneurysm (Table 2-8), the acquired color changes on DSA aneurysms with wide necks are $\geq 4 \mathrm{~mm}$, width $\geq 5 \mathrm{~mm}$ dome, and dome height $\geq 12 \mathrm{~mm}$. This suggests that color changes, when processed by MATLAB DSA, are strongly influenced by the size of the aneurysm morphology (Table 3-5). This is consistent with the factors influencing the occurrence of turbulence in the blood flow within the aneurysm according to Reynold's equation. The first stage in processing statistical data is the variable normality test using the Kolmogorov-Smirnov and the Shapiro-Wilk test; the variable normality test showed that the sample distribution was not normal, so another test was done using Spearman's correlation statistics. Based on Spearman's correlations (Table 6), there was a significant correlation between high-value dome, dome-neck ratio, and the aspect ratio with color changes on the DSA that had been processed using MATLAB $(\mathrm{p}<0.01)$.

\section{DISCUSSIONS}

One study conducted between January 1996 - January 2003 in Tangerang, Indonesia, consisted of 74 cases of intracranial aneurysm and 73 cases underwent early surgery for aneurysm clipping. The study took initial condition (international cooperative study or ICS grading), age, size and location of aneurysms by using an angiogram and the discharge outcome using Glasgow Outcome Scale a.k.a GOS). One patient refused to receive surgery and died because of re-bleeding. There were 73 cases with an average age of 41.4 years old (23-68) and $71.2 \%$ of patients were under the age of 50 . Most aneurysms were medium size $(49.3 \%)$ and the most common aneurysm location was in the posterior communicating artery $(34.2 \%)$, followed by the anterior communicating artery (30.1\%). Forty-nine patients with grades between 1-3 on the ICS showed excellent or good results (GOS 4-5) (92.5\%), compared to 7 patients 
Table 4 Statistic description on aneurysm's Neck wide, Dome wide and Dome height based on its location

\begin{tabular}{lccc}
\hline Variables & Neck Wide & Dome Wide & Dome Height \\
\hline ACA & $3.30 \pm 1.20$ & $3.87 \pm 1.06$ & $3.58 \pm 0.22$ \\
AComA & $5.95 \pm 6.13$ & $8.60 \pm 7.85$ & $10.13 \pm 12.71$ \\
AChoA & $2.07 \pm 0.05$ & $2.32 \pm 0.26$ & $2.92 \pm 0.06$ \\
ICA & $2.95 \pm 1.15$ & $4.20 \pm 1.96$ & $4.44 \pm 3.64$ \\
MCA & $4.04 \pm 2.37$ & $5.63 \pm 4.20$ & $5.51 \pm 3.70$ \\
PComA & $4.74 \pm 2.06$ & $5.73 \pm 1.45$ & $4.13 \pm 1.05$ \\
VA & $6.95 \pm 3.89$ & $7.64 \pm 5.47$ & $8.55 \pm 8.98$ \\
\hline
\end{tabular}

Note: Anterior Cerebral Artery (ACA), Anterior Communicating Artery (AComA), Anterior Chroidal Artery (AChoA), Internal Carotid Artery (ICA), Medial Cerebral Artery (MCA), Posterior Communicating Artery (PComA), Vertebral Artery

Table 5 Correlation among Neck Wide, Dome Wide, Dome Height, Dome Neck Ratio, and Aspect Ratio with DSA color changing on software MATLAB

\begin{tabular}{|c|c|c|c|c|}
\hline & & \multicolumn{2}{|c|}{ DSA Color Changing } & \multirow[b]{2}{*}{ Total } \\
\hline & & No & Yes & \\
\hline \multirow[t]{2}{*}{ Neck Wide } & $<4 \mathrm{~mm}$ & 3 & 16 & 19 \\
\hline & $\geq 4 \mathrm{~mm}$ & 0 & 8 & 8 \\
\hline Total & & 3 & 24 & 27 \\
\hline \multirow[t]{7}{*}{ Dome Wide } & $<2,50$ & 1 & 5 & 6 \\
\hline & $2,50-4,99$ & 2 & 11 & 13 \\
\hline & $5,00-7,49$ & 0 & 3 & 3 \\
\hline & $7,50-9,99$ & 0 & 2 & 2 \\
\hline & $10,00-12,49$ & 0 & 1 & 1 \\
\hline & $12,50-14,99$ & 0 & 1 & 1 \\
\hline & $>17,50$ & 0 & 1 & 1 \\
\hline Total & & 3 & 24 & 27 \\
\hline \multirow[t]{3}{*}{ Dome Height } & $<12 \mathrm{~mm}$ & 3 & 20 & 23 \\
\hline & $12-25 \mathrm{~mm}$ & 0 & 3 & 3 \\
\hline & $>25 \mathrm{~mm}$ & 0 & 1 & 1 \\
\hline \multirow[t]{2}{*}{ Dome Neck Ratio } & $<2$ & 3 & 20 & 23 \\
\hline & $>2$ & 0 & 4 & 4 \\
\hline Total & & 3 & 24 & 27 \\
\hline \multirow[t]{5}{*}{ Aspect Ratio } & $<0.50$ & 2 & 0 & 2 \\
\hline & $0.50-0.99$ & 1 & 5 & 6 \\
\hline & $1.00-1.49$ & 0 & 8 & 8 \\
\hline & $1.50-1.99$ & 0 & 7 & 7 \\
\hline & $>2.00$ & 0 & 4 & 4 \\
\hline Total & & 3 & 24 & 27 \\
\hline
\end{tabular}

Table 6 Spearman's statistic value on Neck Wide, Dome Wide, Dome Height, Dome-Neck Ratio and Aspect Ratio based on DSA color changing

\begin{tabular}{lccccc}
\hline & $\begin{array}{c}\text { Neck } \\
\text { Wide }\end{array}$ & $\begin{array}{c}\text { Dome } \\
\text { Wide }\end{array}$ & $\begin{array}{c}\text { Dome } \\
\text { Height }\end{array}$ & $\begin{array}{c}\text { Dome Neck } \\
\text { Ratio }\end{array}$ & $\begin{array}{c}\text { Aspect } \\
\text { Ratio }\end{array}$ \\
\hline Correlation Coefficient & 0.09 & -0.32 & -0.55 & -0.5 & -0.53 \\
Sig. (2-tailed) & 0.652 & 0.106 & 0.003 & 0.008 & 0.004 \\
N & 27 & 27 & 27 & 27 & 27 \\
\hline
\end{tabular}

(35\%) that had a grade $4-5$ on the ICS. The overall mortality rate was $13.6 \%$ (10 patients), and $90 \%$ of the deaths came from the later groups (9 patients). Nine patients died from pneumonia and vasospasm and one patient died from intracranial infection. Results were generally favorable, with a mortality rate of $1.4 \%$ for the cases with an ICS grade between 1-3 and 92.5\% of patients showed excellent or good outcomes. ${ }^{11}$

The study subjects were 14 women (52\%) and 13 men (48\%), which is consistent with the data on intracranial aneurysm incidence that show that prevalence in women is higher than that in men by a ratio of 3:2. ${ }^{12}$ However, regarding the incidence related to the patient's age, at $<40$ years of age, incidence of aneurysm was higher in male patients. ${ }^{12}$ In our study, there were 13 people (48\%) in the 50-59 year age range. This is consistent with the global literature, in which the incidence was the highest in the age group of 30-60 years. ${ }^{13}$ In global epidemiological data, the locations of the aneurysm include the internal carotid artery $(26 \%)$ followed by the middle cerebral artery $(23 \%)$ and the anterior cerebral artery (15\%).

Advances in neuroimaging techniques provide an alternative method that helps clinicians to diagnose aneurysms more easily. Non-invasive methods, such as a computed tomographic angiography (CTA) and magnetic resonance angiography (MRA), allow the detection of 3D characteristics of aneurysms in order to evaluate the aneurysm morphology. A CT-scan or an MRI can also provide important information in planning for the operation (what kind of information). However, aneurysms with minor bleeding can be detected by using a non-invasive method; by combining several supporting diagnoses, $97 \%$ of cases can be identified exactly. ${ }^{14}$ The technique that is most often used to diagnose intracranial aneurysm is a conventional cerebral angiography (CTA or MRA). Until now, the diagnosis of intracranial aneurysms is still done manually by direct observation of its corresponding medical imaging photo. In addition, medical photographs are also still presented in monochrome form, which takes time to observe. ${ }^{14}$ 
In the statistical test using Spearman's test, we found significant correlations between high dome, dome-neck ratio, and the aspect ratio $(\mathrm{p}<0.01)$ with the color changes in the DSA, which is processed using MATLAB software. This shows that there is a significant correlation between the size of the high dome, the dome-neck ratio, and the aspect ratio with the intra-aneurysm turbulence, causing a delay in the emptying time of contrast agent intra-aneurysm. Furthermore, it is captured in the DSA data as an increase in density or pixel gray. These data show that the results are in agreement with a study done by Ferguson (1970) and Parlea et al. (1999) who managed to make a model of intra-aneurysm turbulence flow on the specific size. ${ }^{15}$ Based on our study, this change in color on the data that was analyzed using MATLAB software, is expected to help clinicians detecting intracranial aneurysms more easily and quicker. In addition, our alternative method would also be able to calculate the intra-aneurysm turbulence flow value to predict its rupture. An ongoing study is underway to validate the equation used to calculate the turbulence flow in patients. However, our attempts to develop and analyze an alternative method had several limitations, such as limitations on the number of samples, the variation of the size of the aneurysm morphology, and time. Therefore, increasing the sample size and investigating each group further is necessary in order to avoid bias calculation and to obtain better results.

\section{CONCLUSIONS}

There is a significant correlation between the size of the aneurysm morphology after the data is processed in MATLAB using the TTP and in combination with TD using the filter MVEF to improve the vessel segmentation by reducing noise in the output process. In addition, this study suggests an alternative method that would facilitate the detection of intracranial aneurysms by visualizing conventional 2D monochrome DSA in the form of a specific color.

\section{CONSENT}

All authors declare that 'written informed consent was obtained from the patient (or other approved parties) for publication of this study and accompanying images.

\section{ETHICAL APPROVAL}

This study protocol was approved by the Faculty of Medicine, Universitas Padjadjaran Review Board, and all research participants gave informed consent.

\section{AUTHORS' CONTRIBUTIONS}

$\mathrm{AF}$ and $\mathrm{AD}$ designed the study. $\mathrm{AF}$ and $\mathrm{WH}$ performed statistical analyses and drafted the manuscript. WH and BRH maintained the study database and contributed to the data analysis. MZA made critical revisions to the manuscript. AF obtained funding of the study. All authors read and approved the final manuscript.

\section{REFERENCES}

1. Linn FH, Rinkel GJ, Algra A, et al. Incidence of subarachnoid haemorrhage: role of region, year and rate of computed tomography: a meta-analysis. Stroke. 1996; 27(4):625-39.

2. Kassell NF, Torner JC, Jane JA, et al. The international cooperative study on timing of aneurysm surgery. Part 2: surgical results. J Neurosurg. 1990;73(1):37-47.

3. Hackett ML, Anderson CS. The Australian Cooperative Research on subarachnoid study group: health outcome 1 year after subarachnoid haemorrhage: an international population based study. Neurology. 2000;55:658-62.

4. Bajamal AH. Stroke Subarachnoid Bleeding: Diagnosis Problem and Management. Disampaikan dalam pidato pengukuhan guru besar ilmu Bedah Saraf FK UNAIR, 16 Januari 2010. Pp 15.

5. Cameron JR, Skofronick JG. Medical Physics. Canada: John Wiley \& Sons. Inc. 1978.

6. Reynolds O. An experimental investigation of the circumstances which determine whether the motion of water shall be direct or sinuous and of the law of resistance in parallel channels. Phil. Trans. R. Soc.1883;174:935-87.

7. Stehbens WE. Histopathology of cerebral aneurysms. Archs Neurol., Chicago 1963;8:277-85.

8. Zakaria H, Kurniawan A, Mengko TLR, Santoso OS. Detection of cerebral aneurysms by using the based parametric color coded of cerebral angiogram. Proceeding: International Conference on Electrical Engineering and Informatics, 17-19 July 2011, Bandung, Indonesia.

9. Menhidratta, A., Knopp, M.V., Zechmann, C.M., et al. 2009. Comparison of Diagnostic Quality and Accuracy in Color-Coded Versus Gray-Scale DCE-MR Imaging Display. Int J Comput Assist Radiol Surg. Vol. 4. pp. 457-62.

10. Strother, C.M., et al. 2010. Parametric Color Coding of Digital Subtraction Angiography. American Journal of Neuroradiology. Vol. 31. pp. 919-24.

11. Johnston SD, Robinson TJ. Subarachnoid haemorrhage: difficulties and diagnosis and treatment. Postgrad Med J. 1998;74(878):743-4.

12. Wahjoepramono EJ, Junus J. Surgical Management on Intracranial Aneurysm. J Kedokter Trisakti 2003;22(2):56-60.

13. Pritz, Michael B. 2003. Subaracnoid Hemorrage Due to Cerebral Aneurysms : Neurological Therapeutics Principles and Practice Volume 1. 48 : 493-503. Martin Dunitz-Taylor and Francis Group.

14. Haberland, Catherine (2007). Clinical neuropathology: text and color atlas ([Online-Ausg.]. ed.). New York: Demos. p. 70. ISBN 978-1-888799-97-2.

15. Ferguson GG. Turbulence in human intracranial saccular aneurysms. J Neurosurg 1970;33:485-497.

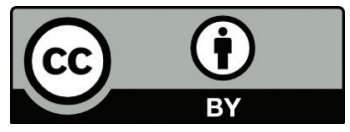

This work is licensed under a Creative Commons Attribution 